\title{
Aggressive Melanoma of Pinna: A Rare Presentation
}

\author{
Chintale Sambhaji G*, Kirdak vilas R, Jatale sonali P, Shaikh $\mathbf{K}$ \\ ENT Department, Muhs nashik university, India
}

*Corresponding author: Dr Chintale sambhaji govind, Associate professor ENT department, Muhs nashik university, Jiius iimsr warudi tq badnapur, Dist. jalna 431202, Maharashtra, India, Tel: 919970323030; Email: drsamchinto@gmail.com

\section{Case Report \\ Volume 2 Issue 1}

Received Date: April 21, 2018

Published Date: May 14, 2018

DOI: $10.23880 /$ ijtps-16000114

\section{Abstract}

Pinna and Skin diseases of pinna are seen in a different condition of medical disorders. Skin specialist, ENT surgeons, general practitioners, general and plastic surgeons are regularly consulted regarding cutaneous lesions on the ear. Here we reported case of melanoma of pinna. A 5 years female child present with pigmented swelling over left side pinna since birth. We excised this melanoma of pinna with primary suturing with advancement of postural skin. In follow up period patient was fine. This type of case report will focus on those diseases where surgical line of management or laser therapy is considered as a treatment of choice.

Keywords: Pinna; Skin diseases; Cerumen; Ear; Periosteum; Lentigo malign melanoma

Abbreviations: UV: Ultraviolet; NM: Nodular Melanoma; SSM: Superficial Spreading Melanoma; LMM: Lentigo Malign Melanoma; SLNB: Sentinel Lymph Node Biopsy.

\section{Introduction}

While examining the skin lesions on the ear, specifically some anatomical characteristic should have been considered. The outer ear consists of the external ear canal and the auricle. Both parts are consisting of elastic cartilage covered with skin. It is tightly attached to the periosteum and poorly vascularized. In contrast the convex aspect of the outer ear has a thicker sub cutis with a stronger layer of subcutaneous fat which causes a certain laxity and displace ability compared to the concave side. The high concentration of Holocaine cacuminal glands in the skin of the external ear canal. The cerumen which is part of wax may mask existing diseases of the skin in the entrance of the external ear canal. In case of a ceruminal obstruction, an adequate assessment of the external auditory meatus should be done only after cleaning, which may damask existing dermatosis. The auricle is susceptible to external environmental factors and trauma. Because of its exposed localization, the ear is mostly affected by the effects of ultraviolet (UV) light and, consequently, to pre-neoplastic and neoplastic skin lesions. Further, pinna has a sound-transmitting function and is located at a visible, esthetically obvious site, drawing considerable attention from the patient. Depending on the site of lesions on the external ear which lead the patient to seek specialist helpand sometime patients himself notice this disease or sometimes noticed by relatives.

When disease present in hidden areas of the ear, specialist consultation may be delayed until very late in the disease process. This is especially true for malignant 


\section{International Journal of Transplantation \& Plastic Surgery}

tumors which may often present at an invasive stage, due to the minimal thickness of the skin compared to other parts of the body. In many cases, we requires a multispecialty approach like skin specialist, ear-nosethroat and surgical collaboration. Below, here we present the most important and frequent skin diseases of the ear which is potentially subject to surgical or laser therapy are described.

\section{Case Report}

A female child patient of 5 yr. age present to otorhinolaryngology department of our institute with pigmented swelling over pinna and surrounding area since 3 years initially this pigmented lesion was small in size then it gradually increased to present size. On systemic examination there were no abnormality in cardiac, respiratory and abdominal systems .on local examination lesion was firm to hard pigmented with nodular formation on surface which gradually increased in size approx. $3.5 \times 3 \mathrm{~cm}$, we have investigated this patient for CBC, HB, KFT, BSL all these report were normal then we decide to excise this lesion with primary suturing under general anesthesia with skin advancement flap (Figure 1).

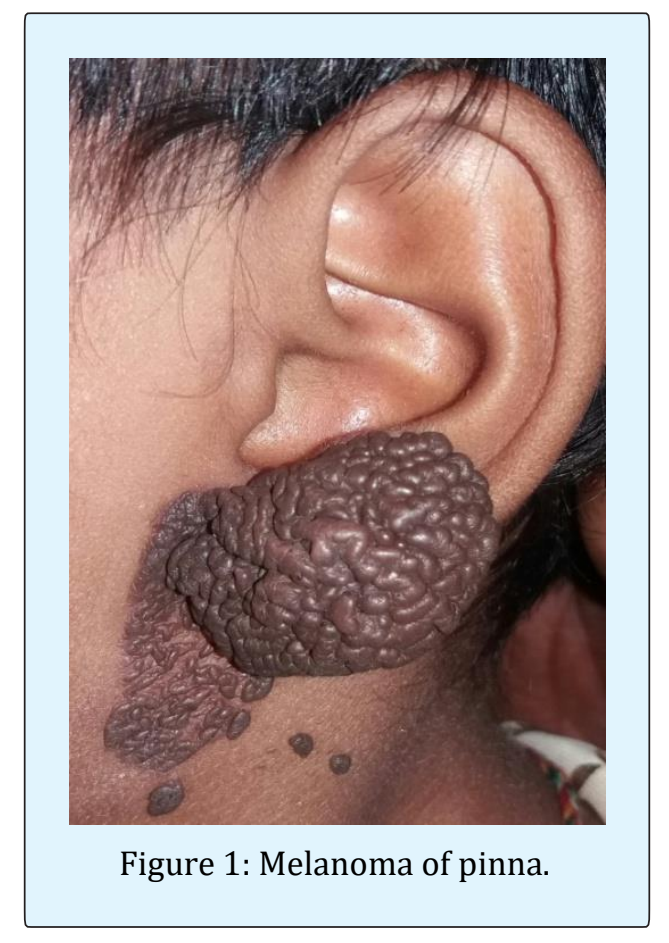

\section{Discussion}

Approximately $20 \%$ of all primary malanomas are presented in the head and neck, of which $7-14 \%$ is located at the pinna specifically at helix and anti-helix. Peripheral parts of the ear are more frequently affected by external environmental factors. Interestingly exact causes do not know but the left ear is more often affected than the right ear. But one most accepted theory for this occurrence is the asymetric UV-dosage in anglosaxon countries with left-hand driven cars. Further, a male exposure to outer environment and predisposition of this type of melanoma rate $61.5-90.5 \%$ is reported in the literature with a predisposition for fair-skinned individuals [1-6]. It can be explained with different hair styles which correlate with UV exposition. The average age for occurrence of this type of disease is 50years. The patients report an asymmetric flat hyperpigmentation or a raised nodular lesion which has changed in color and size.. The three most described subtypes of melanoma are the superficial spreading melanoma, the nodular melanoma and the lentigno maligna melanoma. Each type has its characteristic growth pattern with a horizontal and a vertical growth phase. All over the body, the superficial spreading melanoma is the most common type. It shows an intermediate radial growth phase before starting to invade the dermis. Superficial spreading melanoma. Dark and flat macule with variegated colors. Its borders are irregular, with indentations and notches.

Lentigo malign melanoma characteristic feature is prolonged radial growth phase. A study by Koh, et al. showed that $8.3 \%$ of all head and neck lentigo malign melanomas occur on the ear [6-7]. It begins as a macular lesion with a variable pigmentation with an uneven irregular border which shows a sudden increase in size, in duration and darkening of the lesion. Out of these three types of melanoma the most aggressive melanoma type is the nodular variant, which is a rapidly growing dark pigmented nodule which invades the dermis early in the disease course. The thin layer of subcutaneous tissue contributes to the distinctive invasiveness and therefore bad prognosis for melanoma on the ear. Key pathologic prognostic features of external ear specifically auricular skin melanomas include the histological subtype, tumor thickness, level of invasion and presence of ulceration. Jahn, et al. showed that age, locality, tumor thickness, histological type, level of invasion and excision margins to be significant risk factors for local recurrence [8]. Prognosis of melanoma of pinna depends on the tumor thickness and Clark level of invasion. Therapy for most of melanoma is a surgical approach and in some instances adjuvant therapy. Recommended excision margins are 10-20 mm for primary nodular melanoma (NM) or superficial spreading melanoma (SSM) and $5 \mathrm{~mm}$ with complete three dimensional histology of excision margins (3D histology) for lentigo malign melanoma (LMM). The 


\section{International Journal of Transplantation \& Plastic Surgery}

World Health Association requires a safety margin of 5 $\mathrm{mm}$ for melanoma in situ and $20 \mathrm{~mm}$ for melanoma which are $>2.1 \mathrm{~mm}$ in vertical thickness. Recent studies have shown that margins $>10 \mathrm{~mm}$ have the lowest risk of recurrence [8]. In recent years the more aggressive surgical approach has changed towards narrower excision margins as it has been shown to have only an effect on the incidence of local recurrence and only little impact on disease specific survival.

Lentigo malignant melanoma has the specially irregular pigmented and bordered, brown to black macule with visible bright to reddish regression zone. The available data for sentinel node sampling do not permit definitive conclusions regarding a prognostic or even therapeutic impact of sentinel lymph node biopsy (SLNB) in patients with melanoma of the ear. Depending on the study material of melanoma of pinna Patients with tumors thicker than $1 \mathrm{~mm}$ are currently undergoing SLNB and should be included in large multicenter studies. In special cases when disease is aggressive and depth is more involved where surgical removal of a lentigo maligna melanoma is not possible, radiation therapy should be considered as an alternative with good results [9]. Unfortunately this tumor is aggressive, with a tendency for spreading to both regional lymph nodes and distant sites.

One third of all patients presenting with auricular melanoma have cervical lymph node involvement. As the correlation between melanoma location and drainage is inconsistent lymphoscintigraphy with sentinel node sampling seems to be the primary method of identifying nodal disease and it should be done for as investigation for melanoma $[10,11]$. However a final evaluation is not possible. Adjuvant therapy includes chemotherapy, immunotherapy, and radiation.

\section{Conclusion}

Skin diseases on the pinna and external aspect of the ear are seen in different medical disorders. Dermatologists, othorhinolaryngologists, general practitioners, general and plastic surgeons are regularly consulted regarding cutaneous lesions on the ear. This type of aggressive melanoma has to be dealt appropriately with in time with proper treatment before it spread to neighboring structure.

\section{References}

1. Byers RM, Smith JL, Russell N, Rosenberg V (1980) Malignant melanoma of the external ear. Review of 102 cases. Am J Surg 140(4): 518-521.

2. Davidsson A, Hellquist HB, Villman $\mathrm{K}$, Westman G (1993) Malignant melanoma of the ear. J Laryngol Otol 107(9): 798-802.

3. Arons MS, Savin RC (1971) Auricular cancer.Some surgical and pathologic considerations. Am J Surg 122(6): 770-776.

4. Pockaj BA, Jaroszewski DE, Dicaudo DJ, Hentz JG, Buchel EW, et al. (2003) Changing surgical therapy for melanoma of the external ear. Ann Surg Oncol 10(6): 689-696.

5. Narayan D, Ariyan S (2001) Surgical considerations in the management of malignant melanoma of the ear. Plast Reconstr Surg 107(1): 20-24.

6. Dost P, Lehnerdt G, Kling R, Wagner SN (2004) Surgical therapy of malignant melanoma of the external ear. HNO 52(1): 33-37.

7. Koh HK, Michalik E, Sober AJ, Lew RA, Day CL, et al. (1984) Lentigo maligna melanoma has no better prognosis than other types of melanoma. J Clin Oncol 2(9): 994-1001.

8. Jahn V, Breuninger H, Garbe C, Moehrle M (2006) Melanoma of the ear: prognostic factors and surgical strategies. Br J Dermatol 154(2): 310-318.

9. Stevenson O, Ahmed I (2005) Lentigo maligna: prognosis and treatment options. Am J Clin Dermatol 6(3): 151-564.

10. Cole MD, Jakowatz J, Evans GR (2003) Evaluation of nodal patterns for melanoma of the ear. Plast Reconstr Surg 112(1): 50-56.

11. Wagner JD, Park HM, Coleman JJ $3^{\text {rd }}$, Love C, Hayes JT (2000) Cervical sentinel lymph node biopsy for melanomas of the head and neck and upper thorax. Arch Otolaryngol Head Neck Surg 126: 313-321. 